\title{
IMPLEMENTATION OF PARAMETERIZED WORK PIECE DEVIATIONS AND MEASUREMENT UNCERTAINTIES INTO PERFORMANT META-MODELS FOR AN IMPROVED TOLERANCE SPECIFICATION
}

\author{
Müller, Andreas Michael (1); Oberleiter, Thomas (2); Willner, Kai (2); Hausotte, Tino (1) \\ 1: Friedrich-Alexander-Universität Erlangen-Nürnberg, Institute of Manufacturing Metrology; 2: \\ Friedrich-Alexander-Universität Erlangen-Nürnberg, Chair of Applied Mechanics
}

\begin{abstract}
Geometrical work piece deviations are unavoidable and directly affect the function and quality of technological products. Tolerance management is regarded as a crucial subtask of the development of technological products, because it ensures the function as well as a sufficient product quality while maintaining reasonable production costs. That means, that geometric tolerances as an essential part of the product description greatly affect the functional capability, manufacturability, mountability, verifiability and the costs of the final product. The research group FOR 2271 was founded to enable the computer-aided specification of tolerances, which meet the requirements of production, assembly, verification and function by close cooperation between the departments responsible for product design, assembly and metrology. The aim of this contribution is to determine the manufacturing process scatter as well as the measurement uncertainty and establish ways and means to include that information into efficient meta-models, ultimately enabling improved and accurate tolerance analyses.
\end{abstract}

Keywords: Tolerance representation and management, Uncertainty, Product modelling / models

Contact:

Müller, Andreas Michael

Friedrich-Alexander-Universität Erlangen-Nürnberg

Institute of Manufacturing Metrology

Germany

andreas.mueller@fmt.fau.de

Cite this article: Müller, A.M., Oberleiter, T., Willner, K., Hausotte, T. (2019) 'Implementation of Parameterized Work Piece Deviations and Measurement Uncertainties into Performant Meta-models for an Improved Tolerance Specification', in Proceedings of the 22nd International Conference on Engineering Design (ICED19), Delft, The Netherlands, 5-8 August 2019. DOI:10.1017/dsi.2019.357 


\section{INTRODUCTION}

Geometrical work piece deviations are unavoidable and directly affect the function and quality of technological products. Therefore, the control of those deviations during the product engineering process is crucial for the creation of efficient products, which satisfy the customers' functional needs and overall performance specifications in order to be successful on the market. Consequently, tolerance management is regarded as a crucial subtask of the development of technological products, because it ensures the function as well as a sufficient product quality while maintaining reasonable production costs. That means that geometric tolerances as an essential part of the product description greatly affect the functional capability, manufacturability, mountability, verifiability and the costs of the final product. Today, tolerances are still assigned during the component design based on expert knowledge or statistical data, but without consideration of the component production and verification. Investigations showed that such an approach could in general be regarded as not expedient, because $70 \%$ of all subsequent constructional amendments of work pieces were attributable to geometric deviations, because the given tolerances could not be manufactured or verified (Ceglarek et al., 2004). Data regarding the causes of work piece deviations related to the manufacturing process is not considered during the definition of tolerance specifications. That means that also the measurement uncertainty of the verification process is not taken into account for the specification of work piece tolerances. The activities, which can manage geometrical deviations (tolerance specification, production preparation, preparation for inspection, design of assembly processes) are performed sequentially and mostly independently of each other. This can lead to unnecessary narrow tolerances, which cause cost intensive production, assembly and verification processes. The research group FOR 2271 (German Research Foundation DFG, 2016) was founded to enable the computer-aided specification of tolerances, which meet the requirements of production, assembly, verification and function by close cooperation between the departments responsible for product design, assembly and metrology. Traditional simulation frameworks (e.g. finite element method) reach certain limitations regarding the computational complexity, if statistical elements (e.g. uncertainties) are included into the models. In order to obtain reasonable run times, meta-models were created by the research group, which were based on a small number of process simulations, which could then be used for an integrated tolerance analysis. The aim of this contribution is to determine the manufacturing process scatter as well as the measurement uncertainty and establish ways and means to include that information into efficient meta-models, ultimately enabling improved and accurate tolerance analyses (Figure 1).

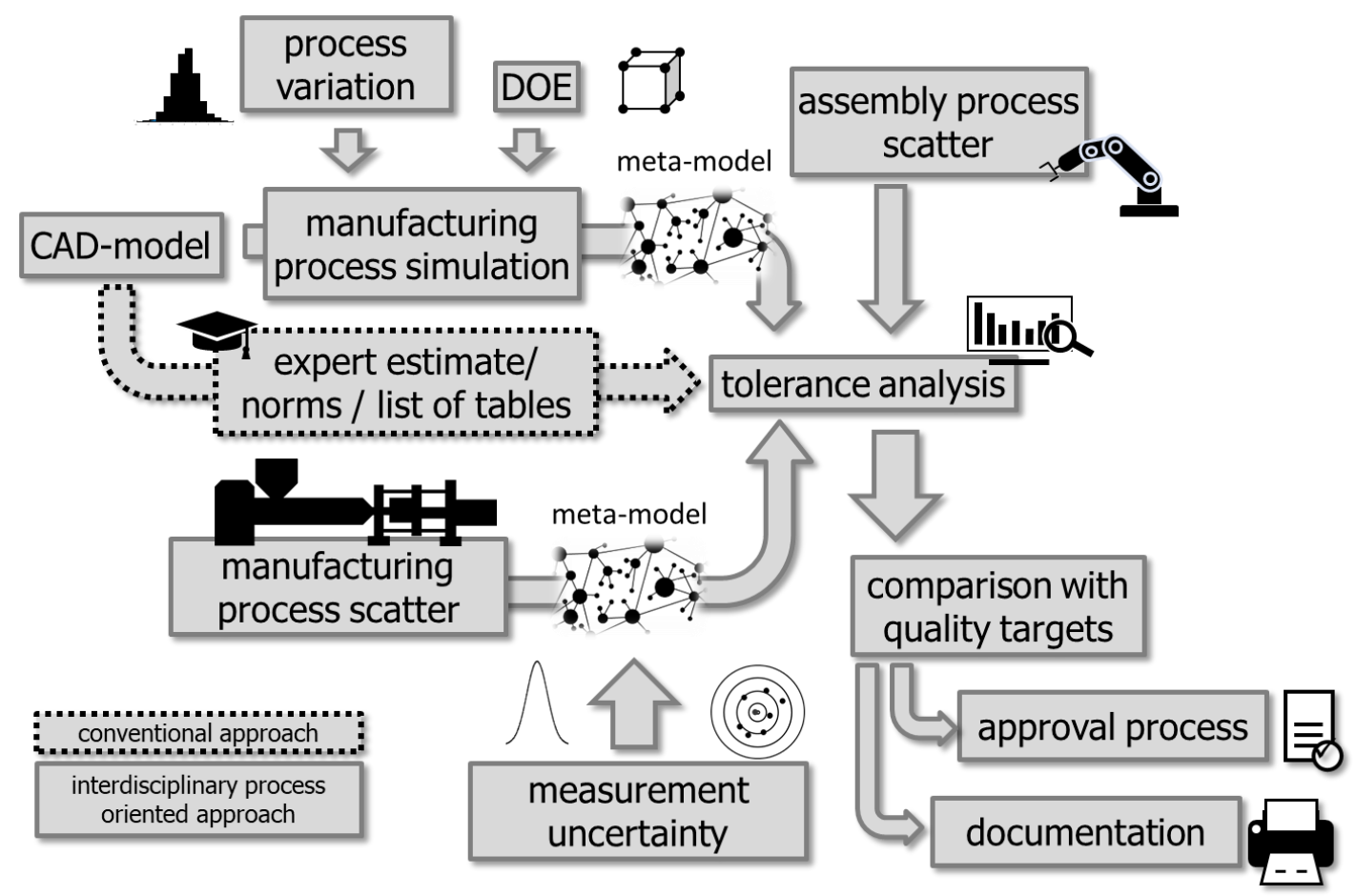

Figure 1: Conventional vs. process oriented approach to a holistic tolerance analysis; according to (Heling et al., 2019) 


\section{REASONS FOR USING META-MODELS FOR TOLERANCE ANALYSIS}

The nominal geometry of a work piece is defined during the design process and is represented as a CAD model. The manufacturing process (in the case of FOR 2271: direct rod extrusion, injection moulding) is characterized by a number of process parameters. If a process is well-known, finite element (FE) simulations can predict the process behaviour for certain input parameter combinations. The subsequent tolerance analysis can then determine the tolerances necessary to ensure the function of the work piece while considering the expected work piece geometry for the input parameter combinations tested with the FE analysis. Common opportunities to quantify uncertainties are Fuzzy sets (Ross, 2017) introduced by Zadeh (Zadeh, 1965) or probability density functions. Depending on the complexity of the FE model, computation times can reach days, even weeks, for one single input parameter combination. This makes the usage of such models very expensive, especially if uncertain parameters are considered. One opportunity to reduce computational time is e.g. a parameter screening as shown in (Heling et al., 2017; Oberleiter et al., 2019). In order to reduce the simulation effort necessary to characterize the manufacturing process, meta-models are created (Forrester et al., 2008). Commonly used meta-models are e.g. Kriging models (Sacks et al., 1989) or response surface methods (Myers et al., 2016). Those models are based on a small number of evaluations of the full model (in this case the FE simulation) and include approximation errors. By adding measurement results including the measurement uncertainty of real prototypes, the meta-model can be improved. A general approach is illustrated in Figure 2.

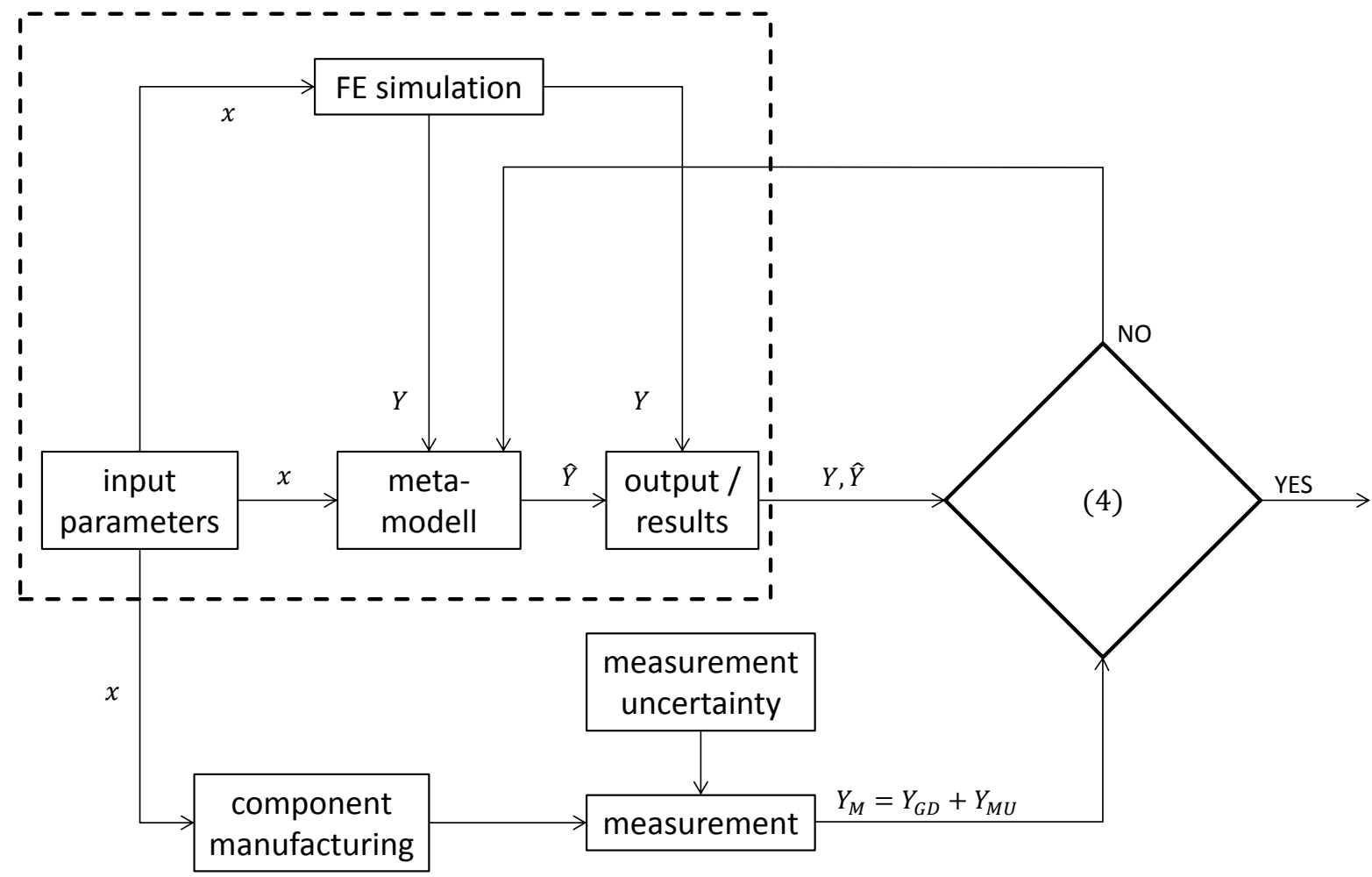

Figure 2: Design of the meta-model (dashed box) implementing different data sources

The meta-model $\hat{f}$ stipulates (1), with $x$ constituting the input parameters and $Y$ the results of the FE simulation, and $\hat{Y}$ the approximated result of the meta-model, thus revealing that the meta-model represents the FE simulation at $x$ with an associated error of $\Delta e r r$.

$$
\hat{f}(x)=\hat{Y}=Y+\Delta e r r
$$

The meta-model can then estimate $\hat{Y}$ for arbitrary input parameters $\hat{x}$, satisfying (2), with $n$ representing the input parameter index and $\overrightarrow{x_{n}}$ the set of FE simulation sampling values for this parameter.

$$
\min \left(\overrightarrow{x_{n}}\right) \leq \hat{x}_{n} \leq \max \left(\overrightarrow{x_{n}}\right)
$$

To increase the significance and robustness of the meta-model, prototypes of the final product are actually manufactured using different manufacturing process input parameters $\left\{x_{j}\right\}$. These prototypes are then validated against the nominal geometry (CAD). The measurement results $Y_{M}$ (3) consist of the 
geometric deviations of the work pieces (and the unknown systematic measurement error) $Y_{G D}$ and the random measurement error $Y_{M U}$.

$$
Y_{M}=Y_{G D}+Y_{M U}
$$

The measurement results for all input parameters $V_{\text {Meas }}=\left\{Y_{M ; j}\right\}$ are then used to calibrate the meta-model by comparison against the corresponding meta model values $V_{\text {Meta }}=\left\{\hat{Y}_{j}\right\}=\left\{\hat{Y}\left(x_{j}\right)\right\}$. In the case of (4), the meta-model is regarded as sufficiently accurate and corresponds well with the measurement results.

$$
\left|V_{\text {Meas }}-V_{\text {Meta }}\right| \leq \varepsilon
$$

Here, $|\cdot|$ represents some suitable metric on the result space. Subsequently the meta-model is adjusted until (4) holds for $V_{\text {Meta }}$. If there are small deviations between the results of the meta-model and the measurement, adjusting the coefficients of the meta-model may be satisfactory. If the deviations of the results are significant, an adjustment of the coefficients is no longer sufficient. Possible strategies are modelling the deviation using another meta-model, which is then superimposed on the existing metamodel. This case can be used if the deviations are of systematic origin (mean value unequal zero). If the deviations are random (mean value equal zero and large amplitude / range), the existing meta-model and the associated FE simulation must be reconsidered and, if necessary, a meta-model based on the real measurements must be created. That means that due to the combination of virtual manufacturing process data (FE simulation) and real measurement data it is possible to significantly increase the prediction fidelity of geometrical parameters necessary for an adjusted accurate tolerance management.

The main reason for utilizing a meta-model is the substantially increased computational performance. That means that the spatial representation of the work piece deviations and the measurement uncertainties should be represented by efficient data structures, which only require little additional computational resources. At the same time, the error introduced while approximating the measurement results in case rather simple geometric models are used, should remain as low as possible to ensure sufficient stability properties of the meta-model.

\section{THE CONCEPT OF THE SINGLE POINT UNCERTAINTY}

The main part of this article deals with possibilities to provide efficiently parameterized representations of the geometric deviations of a work piece and the occurring local measurement uncertainties without introducing additional errors of relevant magnitude caused by the parameterization. Source of the metrological data are repeated measurements of a work piece of interest under supervised and constant conditions. The number of measurement repetitions should be high enough to be statistically significant while avoiding unnecessarily expensive measurement efforts. In case of industrial X-ray computed tomography (CT) measurements, as used in this contribution, a number of 20 repetitions is recommended (VDI/VDE, 2015). The single point uncertainty describes a framework to sample multiple measured surfaces with respect to their common nominal geometry and subsequently statistically evaluate the calculated local geometry deviations. The nominal geometry is used instead of a reference geometry (true quantity values or conventional quantity values of the measured geometry (Brinkmann, 2012)), because the latter is generally unknown if (like for any measurement) the used measurement system is not a reference measurement procedure (Brinkmann, 2012). The reference geometry can be determined using a reference measurement procedure. All surfaces are processed using a triangulated representation of the geometries in the STL format (3D Systems, 1989; Chakravorty, 2018). During the first step of the framework, the measured surfaces are aligned with the nominal geometry (triangulated CAD) by performing a geometrical registration routine, which determines the required affine transformation by iterative minimization of a suitable error function. Commonly used registration algorithms are based on the Iterative Closest Point (ICP) approach, which was proposed almost at the same time by (Chen and Medioni, 1991) and (Besl and McKay, 1992) independently of each other. The registrations in the context of this contribution were performed using the software VGStudio Max 3.2 (Volume Graphics GmbH, 2018). After the registration, there are different sampling strategies possible to determine the spatial relationships between the surfaces (Figure 3). It is important that the triangles, which define the surface of the nominal geometry, have homogeneous sizes over the whole surface, because the triangle edge points define the sampling points $S P_{k}$. The resulting sets of distances $\left(d^{k}=d_{i}^{k}=\left\{d_{1}^{k}, d_{2}^{k}, \ldots, d_{n}^{k}\right\}\right)$ are assigned to their respective nominal surface points $S P_{k}$, which effectively maps the computed distances onto the nominal geometry. The local random measurement error (precision of the measurement system) can then be estimated by the 
determination of the standard deviation of the local intersection distances $d^{k}$. If the exact geometry of the measurement object is unknown, which means that no reference measurement is available, the mean values of the intersection distances $d^{k}$ consist of the local work piece deviations and the local (unknown) systematic measurement error. Known systematic measurement errors must be corrected (International Organization for Standardization, 2008). If the unknown local systematic error of the measurement device is suspected to be large in comparison with the detected local geometric deviations of the work piece, additional local calibration measurements can be performed to determine the reference geometry and thus separating both contributions. The sampling strategies "shortest distance" and "normal vector" (the latter method was firstly introduced according to the authors' knowledge in that context in (Fleßner et al., 2016)), (Figure 3, middle and right) result in very similar dimensional parameters, with the former method exhibiting superior computational run time properties (Müller and Hausotte, 2019a). The third variant sketched "nearest neighbour" neglects the connectivity of vertices into triangles by only considering the nearest vertex, which causes the results to be heavily dependent on the triangle mesh properties, thus rendering the method unsuitable for the following investigations. Finally, the three methods vary considerably regarding the computational requirements, with "nearest neighbour" being the fastest method and "normal vector" being the slowest method.

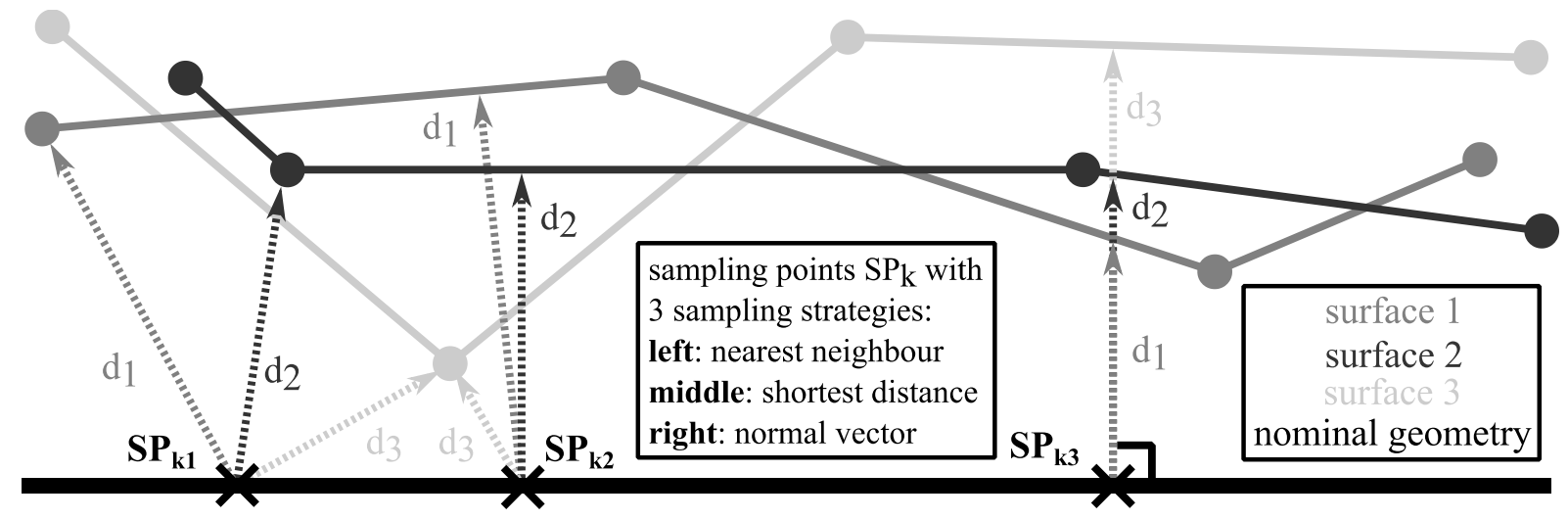

Figure 3: Different sampling strategies for determination of the single point uncertainty

The exact knowledge of the single point uncertainty of a measurement series can greatly influence the quality of the subsequent measurement data evaluations. The processing of single point uncertainties as weighting factors in geometry element fitting routines can lead to a more accurate determination of geometry element properties in dimensional metrology (Müller and Hausotte, 2019b). For CT, the visual evaluation of the locally varying uncertainties directly gives insight into the underlying X-ray penetration lengths (because they directly affect the single point noise due to the impaired signal to noise properties of the recorded projections). The method was successfully used to correct systematic measurement errors in CT measurements by determining the systematic measurement error (here represented by the mean value of $d^{k}$ ) of a simulated measurement series using the virtual CT system from (Wohlgemuth et al., 2018) and subsequently correcting the faulty contributions (Müller et al., 2018). It was shown in (Müller and Hausotte, 2018) that the weighted data fusion of surface data sets using locally defined quality parameters could lead to a better measurement result. This also defines a valid usage scenario for the single point uncertainty framework in case the required large measurement expenses for repeated measurements can be justified for certain applications.

The measurement series used for demonstration purposes of the implemented sampling strategies consists of 20 repeated measurements of a polymer (Polyoxymethylene, POM) gear wheel (straight toothed, 39 teeth, thickness $6 \mathrm{~mm}$ ) with a form tip diameter of ca. $40 \mathrm{~mm}$, which were performed on the industrial CT system "Zeiss Metrotom 1500". The measurement object was set up in such way, that the rotation axis of the rotatory stage of the CT system was aligned parallel with respect to the rotation axis of the gear wheel. The surface determination from the raw volume data was performed using VGStudio Max 3.2 using automatic material definition and iterative surface determination (Volume Graphics GmbH, 2018). The industrial CT system was chosen for this proof of concept, because it enables an extensive and complete capture of the geometry of the work piece featuring a very high surface point density. It is expected, that in case of polymer work pieces the unknown systematic measurement error is small compared to the deviations of the work piece. The CT system was periodically calibrated using the procedure described in (VDI/VDE, 2011). If the required measurement durations and the reduced measurement point density can 
be justified, it is also possible to use a coordinate measurement machine (CMM) with improved measurement uncertainty characteristics by probing a number of line profiles per gear wheel tooth.

The goal of the target meta-model is to represent the complete characteristics of functional surfaces like the contact area of gear wheel teeth. Figure 4 shows the precision (local standard deviation of distances $d^{k}$ ) of the repeated CT measurements, which varies depending, inter alia, on the material penetration lengths (see tooth flanks). For this demonstration, the sampling strategy "shortest distance" was used. Edge regions are characterized by a larger uncertainty value. The nominal geometry was processed to feature normally distributed triangle edge lengths of $(58.9 \pm 20.1) \mu \mathrm{m}$ resulting in about 3.4 million triangles in the mesh (Müller and Hausotte, 2019a). The results are dependent on the specific measurement task and system settings. Goal with regard to the meta-model and the subsequent tolerance analysis is to describe the spatial distribution of the precision and the systematic work piece deviations.

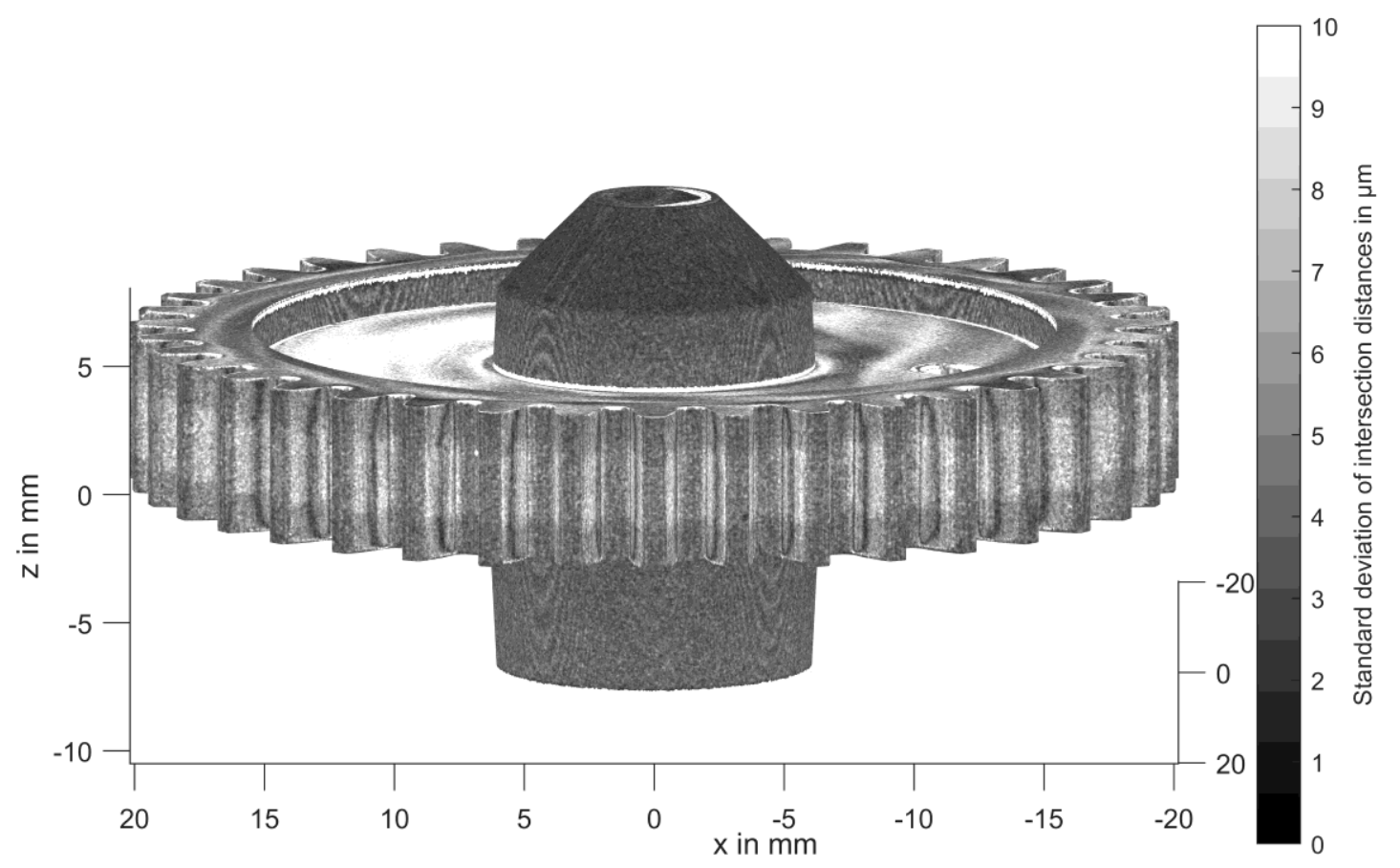

Figure 4: Precision of a polymer gear wheel measurement series

\section{PARAMETERIZATION OF THE GEOMETRY AND THE UNCERTAINTY}

A gear wheel consists of many functional surfaces, which ensure the function during operation as intended by the designer. These surfaces are the cylindrical hole defining the rotation axis in combination with the surface areas in contact with the partner gear wheel during coupling. From the standpoint of an improved tolerance analysis, only these surface areas are of interest, which means that only the work piece deviations and local measurement uncertainties of those areas need to be approximated. For the following demonstration purposes the deviations for each tooth in the region defined by the profile total deviation evaluation field specified in (International Organization for Standardization, 2018) were considered. The general approach for the parameterization is visualized in Figure 5: The nominal geometry of the gear wheel (see also Figure 4) is aligned in such a way that the xz-plane divides the profile of a tooth. That means that the left profile part and the right profile part are aligned symmetrically with respect to the xz-plane. Subsequently, the data points $S P_{k}$ including the associated deviations $d^{k}$ of that specific gear wheel tooth within the profile total deviation evaluation range can be approximated by a combination of suitable functions for each gear wheel flank. After that, the nominal geometry (consisting of surface points $S P_{k}$ ) is rotated by one gear wheel pitch and the evaluation of the next tooth is performed within the same coordinate system. This ensures the comparability of the parameterization results of all teeth with respect to each other and with respect to different measurement series with the same nominal geometry. The overall parameterization result $f_{\text {total }}$ (5) for a specific tooth $n$ and flank $s$ is defined by the combination of three different functions, thus defining a specific confidence band for the expected deviations and uncertainties. The function $f_{\text {total }}(5)$ consists of two functions $f_{\text {total }}^{u p}$ and $f_{\text {total }}^{\text {down }}$, which define the upper and the lower limit of the uncertainty 
corridor (see also Figure 6). Each of those two functions feature three different parts, which approximate the nominal geometry (6), the geometric deviations including the unknown systematic measurement error (7) and the local precision of the measurement setup (8). Each of those functions is defined in the same coordinate system frame (Figure 5), which is dependent on the tooth index $n$ and the flank $s$.

$$
\begin{aligned}
& f_{\text {total }}=\left\{f_{\text {total }}^{u p}, f_{\text {total }}^{\text {down }}\right\}, f_{\text {total }}^{u p}=f_{\text {geo }}+f_{\text {sys }}+f_{\text {rand }}, f_{\text {total }}^{\text {down }}=f_{\text {geo }}+f_{\text {sys }}-f_{\text {rand }} \\
& y \approx f(x, z, n, s)=f_{\text {geo }}, \text { withs } \in\{\text { “left","right" }\} \\
& \operatorname{mean}\left(d^{k}\right) \approx f(x, z, n, s)=f_{\text {sys }} \\
& \operatorname{std}\left(d^{k}\right) \approx f(x, z, n, s)=f_{\text {rand }}
\end{aligned}
$$

This approach results in $2 m$ ( $m$ equals number of gear wheel teeth) functions $f_{\text {geo }}, f_{s y s}, f_{\text {rand }}$ for each gear wheel, thus approximating the measurement series in the profile total deviation range. The approximation results for query coordinates $\left[x_{q} y_{q} z_{q}\right]$ satisfying the profile total deviation definition range (9) (gear wheel rotation axis equals z-axis) are then generally accessible through a map $T(10)$.

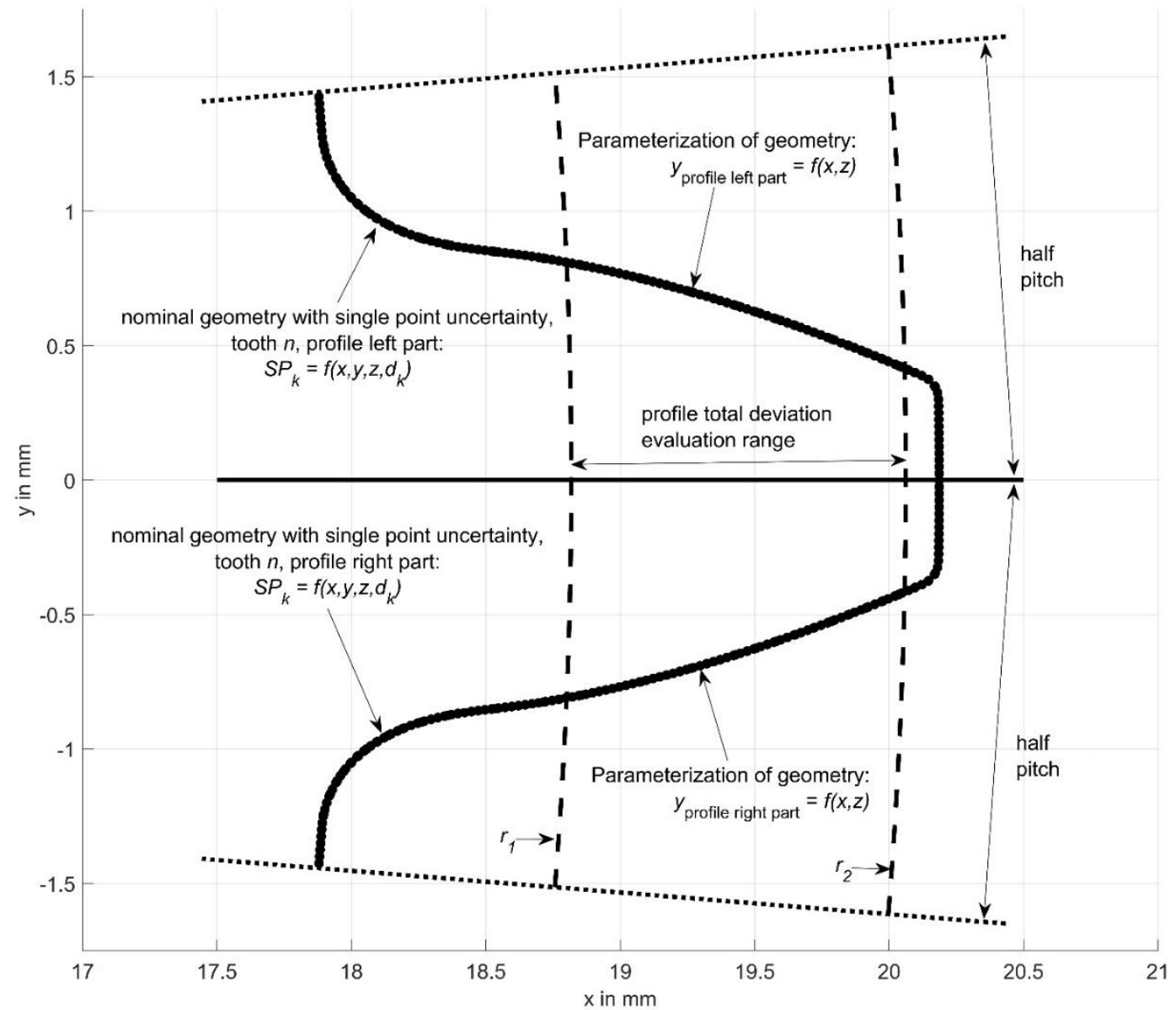

Figure 5: Definition of coordinate systems for gear wheel parameterization inside profile total deviation evaluation range

$$
\begin{aligned}
& {\left[x_{q}^{2}+y_{q}{ }^{2} \geq r_{1}^{2}\right] \wedge\left[x_{q}{ }^{2}+y_{q}^{2} \leq r_{2}^{2}\right] \wedge\left[z_{q} \geq z_{\text {min }} \wedge z_{q} \leq z_{\max }\right]} \\
& T:\left[x_{q} y_{q} z_{q}\right] \mapsto x, z, n s
\end{aligned}
$$

The functions $f$ can be represented by different models, which influence the parameterization result with respect to complexity and approximation accuracy. In this context, the following four different parameterization models were considered:

1. Linear interpolation (The MathWorks Inc., 2018b)

2. Local Regression Smoothing using "lowess" model with linear polynomial (The MathWorks Inc., 2018a) 
3. Local Regression Smoothing using "loess" model with quadratic polynomial (The MathWorks Inc., 2018a)

4. Absolute polynomial models using "poly55" model (The MathWorks Inc., 2018c)

The linear interpolation model was introduced as the "ground truth" model, because the residuals are zero at the grid points per definition (within floating point accuracy). Even though the linear interpolation model represents the optimal solution for many usage scenarios, the possibly existing constraints of the meta-model regarding the smoothness and steadiness of the included data for stability reasons render the examination of alternative parameterization methods necessary. All fit routines (parameterization) were performed using MATLAB using the coordinate system as introduced in Figure 5. The option "Normalize" was activated to centre and scale the data during the computations; all other settings were used in the "default" state without any changes.

\section{PARAMETERIZATION RESULTS AND COMPARISON OF RESIDUALS}

Figure 6 shows the parameterization result $f_{\text {total }}(5)$ for the left flank of tooth 10 using a polynomial model. The two curves limit the uncertainty corridor of the measurement series for that specific gear wheel flank within the profile total deviation evaluation range as defined in (International Organization for Standardization, 2018). It is observable that the random measurement error reached relevant magnitudes compared to the systematic work piece deviations (incl. unknown systematic measurement error). That means that it is important to observe both parameters in order to construct a robust metamodel. The prediction fidelity can be extended by the introduction of a coverage factor (here $k=1$ ) for the uncertainty, as defined in (International Organization for Standardization, 2008).

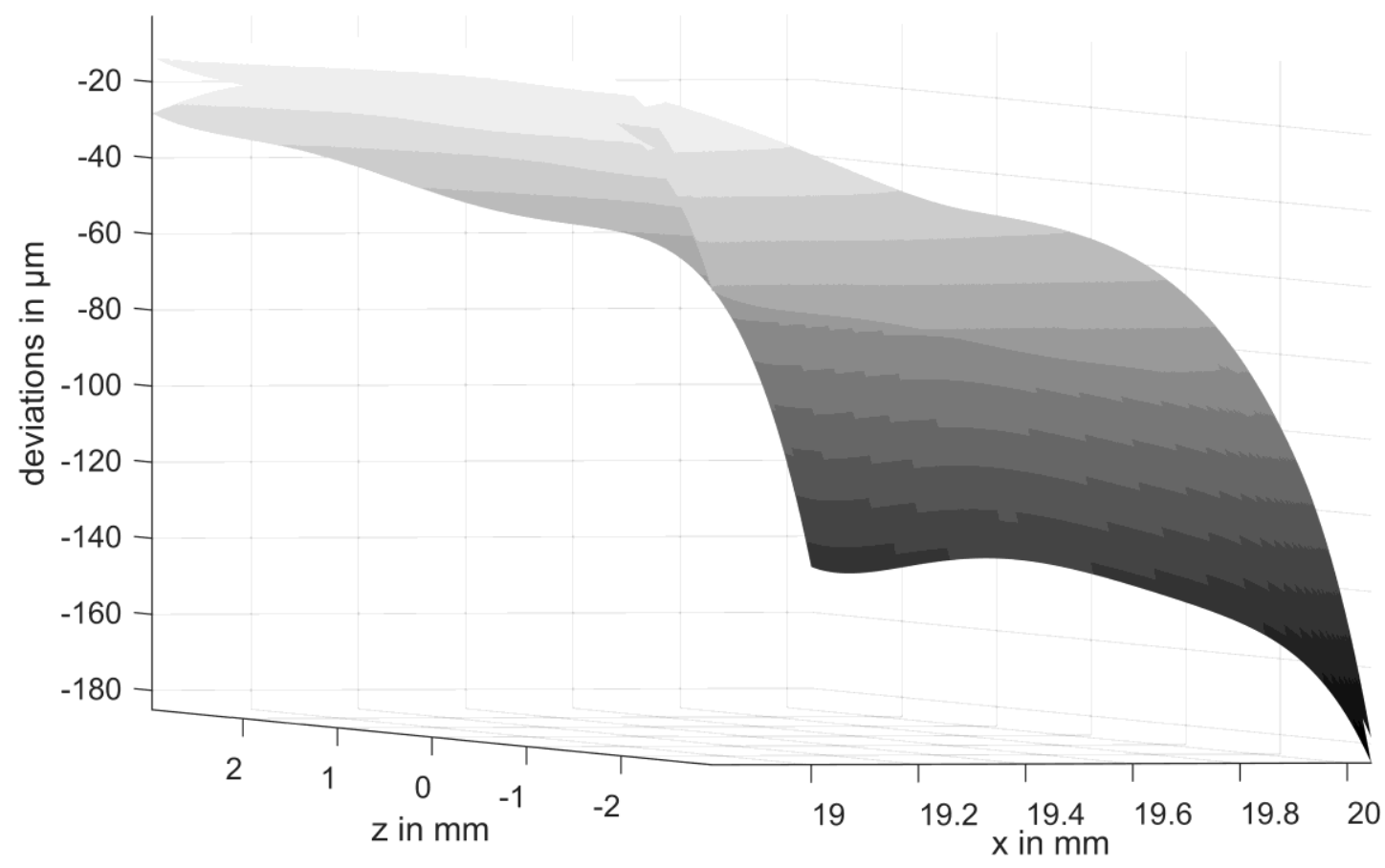

Figure 6: Parameterization result $f_{\text {total }}$ of the mean deviations \pm the computed local precision of left flank of tooth 10 with polynomial fit model "poly55", deviations in grayscale

The examined fit models differ from each other with regard to their mathematical complexity and subsequently their ability to approximate the given measurement data. The three different parts of the measurement data (nominal geometry (6), systematic deviations / measurement error (7) and precision of the measurement system (8)) also exhibit different characteristics regarding the magnitude and distribution of values. Table 1 shows the comparison of the resulting root mean squared errors (RMSE) after convergence of the respective fit algorithms. The shown values represent the mean value and the standard deviation value of the fits of all teeth and both left and right profiles. The polynomial model and the quadratic local regression model show very similar results, while the linear local regression model performs slightly worse. The RMSE values for the linear interpolant are per definition zero. 


\section{Table 1: Comparison of fit RMSE values for individual models and datasets}

\author{
Fit RMSE over all teeth \\ mean \pm std. dev. in $\mu \mathrm{m}$ \\ polynomial \\ local regression linear \\ local regression quad. \\ linear interpolant
}

\author{
function (6): \\ nominal geometry \\ $0.056 \pm 0.013$ \\ $1.708 \pm 0.033$ \\ $0.056 \pm 0.012$ \\ $0 \pm 0$
}

\author{
function (7): \\ mean deviations \\ $4.525 \pm 0.866$ \\ $8.466 \pm 0.909$ \\ $4.831 \pm 0.901$ \\ $0 \pm 0$
}
function (8):
std. dev. of deviations
$1.277 \pm 0.064$
$1.310 \pm 0.061$
$1.239 \pm 0.061$
$0 \pm 0$

\section{CONCLUSION}

In this article, a universal methodology to include data from repeated measurements into subsequent meta-models was presented. The comparison of a measurement series with respect to its nominal geometry was performed using the single point uncertainty method, which was already implemented and used successfully in different metrological contexts. The parameterization of the nominal geometry within the total profile deviation evaluation range and the corresponding systematic work piece deviations in combination with the measurement uncertainties was done using three different functions for each flank of each gear wheel tooth. Four different parameterization models were implemented and compared against each other. The results showed similar RMSE for polynomial and quadratic local regression models, thus making the polynomial model most suited in that context, if computation run time is also considered. Future research efforts could expand the parameterization models with robust variants of the examined models.

The presented use case, which is implementing metrological data into a meta-model to optimize subsequently performed tolerance analysis, enables a design team to define more robust tolerances and thus avoiding unnecessary prototyping and correction loops.

\section{REFERENCES}

3D Systems, I. (1989), StereoLithography Interface Specification.

Besl, P.J. and McKay, N.D. (1992), "A Method for Registration of 3-D Shapes", IEEE Transactions on Pattern Analysis and Machine Intelligence, Vol. 14 No. 2, pp. 239-256.

Brinkmann, B. (2012), International vocbulary of metrology: Basic and general concepts and associated terms(VIM) German-English version ISO/IEC Guide 99:2007, Corrected version 2012, Beuth Wissen, 4., Aufl., Beuth, Berlin.

Ceglarek, D., Huang, W., Zhou, S., Ding, Y., Kumar, R. and Zhou, Y. (2004), "Time-Based Competition in Multistage Manufacturing: Stream-of-Variation Analysis (SOVA) Methodology-Review”, International Journal of Flexible Manufacturing Systems, Vol. 16 No. 1, pp. 11-44.

Chakravorty, D. (2018), Standard Tessellation Language. STL File Format (3D Printing) - Simply Explained, Available at: https://all3dp.com/what-is-stl-file-format-extension-3d-printing/ (accessed 21 November 2018).

Chen, Y. and Medioni, G. (1991), "Object modeling by registration of multiple range images", Proceedings IEEE International Conference on Robotics and Automation, Vol. 3, pp. 2724-2729.

Fleßner, M., Müller, A.M., Götz, D., Helmecke, E. and Hausotte, T. (2016), Assessment of the single point uncertainty of dimensional CT measurements, Available at: https://www.ndt.net/article/ctc2016/papers/ICT2016_paper_id48.pdf.

Forrester, A.I.J., Sóbester, A. and Keane, A.J. (2008), Engineering design via surrogate modelling: A practical guide, 1st ed., Wiley, Chichester.

German Research Foundation DFG (2016), FOR 2271. Prozessorientiertes Toleranzmanagement mit virtuellen Absicherungsmethoden, Available at: http://gepris.dfg.de/gepris/projekt/260682773 (accessed 7 November 2018).

Heling, B., Oberleiter, T., Rohrmoser, A., Kiener, C., Schleich, B., Hagenah, H., Merklein, M., Willner, K. and Wartzack, S. (2019), "A concept for process-oriented interdisciplinary tolerance management considering production-specific deviations. submitted contribution", Proceedings of the International Conference on Engineering Design, ICED.

Heling, B., Oberleiter, T., Schleich, B., Willner, K. and Wartzack, S. (2017), "Comparison of Different Sensitivity Analysis Methods in the Context of Dimensional Management", in Volume 2: Advanced Manufacturing 3, V002T02A102.

International Organization for Standardization (2008), (JCGM/WG1/100): Uncertainty of measurement -- Part 3: Guide to the expression of uncertainty in measurement (GUM:1995), 17.020 Metrology and measurement in general No. ISO/IEC Guide 98-3:2008 (accessed 21 November 2018).

International Organization for Standardization (2018), Cylindrical gears - ISO system of flank tolerance classification-Part 1: Definitions and allowable values of deviations relevant to flanks of gear teeth (ISO 1328-1:2013), ICS 21.200 No. ISO 1328-1:2013 (accessed 20 November 2018). 
Müller, A.M. and Hausotte, T. (2018), "Data fusion of surface data sets of X-ray computed tomography measurements using locally determined surface quality values", Journal of Sensors and Sensor Systems, Vol. 7 No. 2, pp. 551-557.

Müller, A.M. and Hausotte, T. (2019a), "Comparison of different measures for the single point uncertainty in industrial X-ray computed tomography", 9th Conference on Industrial Computed Tomography, e-Journal of Nondestructive Testing.

Müller, A.M. and Hausotte, T. (2019b), "Utilization of single point uncertainties for geometry element regression analysis in dimensional X-ray computed tomography", 9th Conference on Industrial Computed Tomography, e-Journal of Nondestructive Testing.

Müller, A.M., Wohlgemuth, F. and Hausotte, T. (2018), "Simulation-based correction of systematic errors for CT measurements", 8th Conference on Industrial Computed Tomography, e-Journal of Nondestructive Testing.

Myers, R.H., Montgomery, D.C. and Anderson-Cook, C.M. (2016), Response surface methodology: Process and product optimization using designed experiments, Wiley series in probability and statistics, 4 edition, Wiley, Hoboken.

Oberleiter, T., Heling, B., Schleich, B., Willner, K. and Wartzack, S. (2019), "Fuzzy Sensitivity Analysis in the Context of Dimensional Management”, ASCE-ASME J. Risk and Uncert. in Engrg. Sys., Part B: Mech. Engrg., Vol. 5 No. 1, 011008-011008-7.

Ross, T.J. (2017), Fuzzy logic with engineering applications, Engineering professional collection, 4 edition, John Wiley \& Sons Inc, Southern Gate, Chichester, West Sussex, United Kingdom.

Sacks, J., Welch, W.J., Mitchell, T.J. and Wynn, H.P. (1989), "Design and Analysis of Computer Experiments", Statistical Science, Vol. 4 No. 4, pp. 409-423.

The MathWorks Inc (2018a), Filtering and Smoothing Data. Local Regression Smoothing, Available at: https://www.mathworks.com/help/curvefit/smoothing-data.html (accessed 20 November 2018).

The MathWorks Inc (2018b), Interpolation Methods. About Interpolation Methods, Available at: https://www.mathworks.com/help/curvefit/interpolation-methods.html (accessed 20 November 2018).

The MathWorks Inc (2018c), Polynomial Models. Defining Polynomial Terms for Polynomial Surface Fits, Available at: https://www.mathworks.com/help/curvefit/polynomial.html (accessed 20 November 2018).

VDI/VDE (2011), Computed tomography in dimensional measurement: Guideline for the application of DIN EN ISO 10360 for coordinate measuring machines with CT sensors, ICS 17.040.30, 19.100, 35.240.80 No. VDI/VDE 2630 Part 1.3, Beuth Verlag GmbH, 10772 Berlin.

VDI/VDE (2015), Computed tomography in dimensional measurement: Determination of the uncertainty of measurement and the test process suitability of coordinate measurement systems with CT sensors, ICS 17.040.20, 19.100, 35.240.80 No. VDI/VDE 2630 Part 2.1, Beuth Verlag GmbH, 10772 Berlin.

Volume Graphics GmbH (2018), VGSTUDIO MAX - Produkte - volumegraphics.com.

Wohlgemuth, F., Müller, A.M. and Hausotte, T. (2018), "Development of a virtual metrological CT for numerical measurement uncertainty determination using aRTist 2", Tm-Technisches Messen, Vol. 85, pp. 728-737.

Zadeh, L.A. (1965), "Fuzzy sets", Information and Control, Vol. 8 No. 3, pp. 338-353.

\section{ACKNOWLEDGMENTS}

The authors would like to thank the German Research Foundation (DFG) for supporting the research project "FOR 2271 process-oriented tolerance management based on virtual computer-aided engineering tools" under grant numbers WI 1181/8-1 and HA 5915/9-1 and for the financial support of the acquisition of the CT system "Zeiss Metrotom 1500" through Grant No. 324672600. The authors thank the Institute of Polymer Technology (LKT, FAU Erlangen-Nuremberg) for providing the polymer gear wheel used for demonstration purposes in this article. 